\title{
NRF2 is overexpressed in ovarian epithelial carcinoma and is regulated by gonadotrophin and sex-steroid hormones
}

\author{
HONG LIAO $^{1}$, QIAN ZHOU ${ }^{1}$, ZHENBO ZHANG ${ }^{2}$, QIANQIAN WANG ${ }^{2}$, \\ YUNYAN SUN ${ }^{2}$, XIAOFANG YI $^{3}$ and YOUJI FENG ${ }^{2}$ \\ ${ }^{1}$ Clinical and Translational Research Center, Shanghai First Maternity and Infant Hospital, Tongji University \\ School of Medicine; ${ }^{2}$ Department of Obstetrics and Gynecology, Shanghai Jiao Tong University Affiliated \\ First People's Hospital; ${ }^{3}$ Obstetrics and Gynecology Hospital of Fudan University, Shanghai, P.R. China
}

Received November 13, 2011; Accepted January 17, 2012

DOI: $10.3892 /$ or.2012.1700

\begin{abstract}
Aberrant nuclear factor-erythroid 2 (NRF2) expression correlates with tumor development. We investigated NRF2 expression in ovarian epithelial carcinoma (OEC), aiming to identify associations with clinicopathological factors, hormones and induced reactive oxygen species (ROS). Immunohistochemical staining for NRF2 expression was performed on 10 benign cystadenomas, 4 boderline tumors and 64 OECs. Western blotting was used to determine the effects of hormones on NRF2 expression in OEC. NRF2 protein was found to be overexpressed in OEC. Gonadotrophins and estrogen induced ROS production in OEC; these hormones also enhanced NRF2 expression. Therefore, aberrant expression may be induced by hormones through ROS signaling. Increased NRF2 expression may be a molecular event in OEC carcinogenesis.
\end{abstract}

\section{Introduction}

Ovarian cancer is the most complicated malignancy of the female reproductive system, and is the fourth most frequent cause of cancer-related death in women. The mortality rate from ovarian cancer is high as a result of occult metastases within the peritoneal cavity and the typically advanced stage at detection, when curative therapy is ineffective. Over $90 \%$ of ovarian cancers are thought to arise in the ovarian surface epithelium. Tumorigenesis has been associated with inflammation, incessant ovulation and persistent stimulation

Correspondence to: Professor Youji Feng, Department of Obstetrics and Gynecology, Shanghai First People's Hospital, Shanghai Jiao Tong University, 100 Haining Road, Shanghai 200080, P.R. China E-mail: fengyj4806@sohu.com

Dr Xiaofang Yi, Obstetrics and Gynecology Hospital of Fudan University, 419 Fang Xie Road, Shanghai 200011, P.R. China E-mail: yi.annie.1@gmail.com

Key words: nuclear factor-erythroid 2, ovarian epithelial carcinoma, gonadotrophin, sex-steroid hormones, reactive oxygen species from high-level gonadotrophins and sex-steroid hormones. Among these etiologies of ovarian epithelial cancer (OEC), hormone-induced tumorigenesis is a popular hypothesis. A growing body of evidence shows a clear trend of decreasing risk with increasing numbers of pregnancies, with the extent of protection being related to the length of the pregnancy and the duration of breast-feeding. There is also a clear decrease in risk with increasing durations of oral contraceptive (OC) use. These protective factors are linked to cyclic hormonal changes. However, the details of the molecular mechanism(s) of these protective effects are not clear.

Recent studies suggest that reactive oxygen species (ROS) play an important role in tumorigenesis. ROS feature in normal cellular function and biological behaviour, including ovulation. However, increased ROS stress is observed in the cancer microenvironment, and excess ROS have been detected in various cancer types. It may be the case that optimal levels of ROS are required for carrying out biological functions, but that excess ROS result in nucleic acid damage, aging, inflammation and cancer. However, whether ROS are involved in OEC development and whether gonadotrophin and sex-steroid hormones could induce ROS remains to be determined.

In response to the harmful effects of excess ROS, cells have developed a system to prevent ROS accumulation in which antioxidant proteins and phase II detoxifying enzymes are activated. The antioxidant genes are coordinately regulated through the antioxidant response element (ARE) in their promoters and the transcription factor nuclear factor-erythroid 2 (NRF2) is the master regulator of ARE-driven genes. Normal levels of NRF2 are kept low by its constant degradation through ubiquitination by Kelch-like ECH-associated protein 1 (KEAP1), a negative regulator of NRF2. Under high ROS levels, NRF2 is liberated from KEAP1 and transactivates ARE-driven gene expression. A recent study indicated that high NRF2 expression levels in gallbladder adenocarcinoma (GC) was associated with shorter overall survival times and concluded that NRF2 expression may be an important factor in identifying a poor prognostic group of GC (1). Jiang et al reported that type II endometrial cancer had elevated NRF2 expression, and that high levels of NRF2 resulted in chemoresistance (2). Previous research on the role of NRF2 in the pathogenesis of OEC has focused largely on chemoresistance, and few studies have addressed the relationship 
between NRF2 expression and OEC clinicopathological factors or prognosis, or its association with hormones. The aims of the present study are therefore to investigate NRF2 expression in OEC tissues, to identify its association with clinicopathological factors, and to further determine whether this is related to gonadatrophin exposure and sex-steroid hormone-induced ROS.

\section{Materials and methods}

Selection of tissues. Seventy-eight tissue samples were studied, with 10 benign cystadenomas used as controls. Sixty-four cases were OEC, including 11 clear cell carcinomas, 9 endometrioid adenocarcinomas, 6 mucinous cystadenocarcinomas and 38 serous cystadenocarcinomas, while the remaining 4 were cases of borderline tumor. The samples were obtained from the Department of Obstetrics and Gynecology of the Shanghai First People's Hospital, affiliated to Shanghai Jiao Tong University. Patients were diagnosed with OEC and had undergone total abdominal hysterectomy and bilateral salpingo-oophorectomy with retroperitoneal lymphadenectomy (radical pelvic and para-aortic lymphadenectomy was performed in all patients), followed by adjuvant chemotherapy with carboplatin/paclitaxel, with residual tumor $<2 \mathrm{~cm}$. None of the patients had received any pre-operative therapy, such as radiotherapy or chemotherapy, before the ovarian cancer tissue sample was taken during surgery. Patient age ranged from 22 to 79 years (median, 54 years). The clinical stage distribution of the OEC cases was as follows: 13 cases at stage I, 16 cases at stage II and 35 cases at stage III. Lymph node metastasis was found in 31 OEC cases. All tissues harvested included 13 cases of grade I, 18 cases of grade II and 22 cases of grade III tumors; clear cell carcinoma was excluded. The staging and grading of tumors were determined in accordance with the International Federation of Gynecology and Obstetrics (FIGO). Pathological diagnoses of ovarian lesions were made by two gynecological pathologists on the basis of WHO classification in a double-blinded manner.

Tissue processing. All the collected ovarian lesion samples were fixed in $10 \%$ buffered formalin and processed using standard methods for paraffin embedding. The samples were cut as $5-\mu \mathrm{m}$ sections for immunohistochemistry and placed on positively charged glass slides. Histological diagnoses were confirmed by review of the original H\&E-stained tissue sections. Approval for the use of these tissues for research purposes was obtained from the Ethics Committee of the Medical College, Shanghai Jiao Tong University, China and written consent was obtained from all patients.

Immunohistochemical staining. Detection of NRF2 expression was performed using a rabbit polyclonal IgG raised against human NRF2. The paraffin sections were dried at $60^{\circ} \mathrm{C}$ for $2 \mathrm{~h}$, then deparaffinized in xylene and rehydrated in a graded series of alcohol solutions. Endogenous peroxidase activity was blocked by a 10 -min treatment with $3.0 \%$ hydrogen peroxide. Slides were then placed in citrate buffer ( $\mathrm{pH}$ 6.0) and brought to boiling for $10 \mathrm{~min}$ in two 5-min sessions in a microwave. They were then incubated for $30 \mathrm{~min}$ with $0.01 \%$ Trixon, then for $20 \mathrm{~min}$ with $5 \%$ bovine serum albumin. Subsequently, the sections were incubated for $2 \mathrm{~h}$ with the anti-NRF2 antibody diluted 1:100, followed by a 50-min incubation with a biotinylated secondary antibody (Dako, Carpinteria, CA, USA). Human endometrial cancer tissue sections were used as positive controls for NRF2 IHC, as previously described $(2,3)$. The primary antibody was omitted in endometrial cancer sections as a negative control.

Evaluation of NRF2 expression. NRF2 expression was assessed using a semi-quantitative method, as previously described (4). The total score was $\mathrm{AxB}$, where A represented the proportion of positive cells ( $\leq 5 \%$ scored $0,6-25 \%$ scored $1,26-50 \%$ scored 2, 51-75\% scored 3 and $>75 \%$ scored 4 ) and B indicated the intensity of staining (none as 0 , weak as 1 , moderate as 2 and strong as 3). Therefore, a total score of 0 indicated no NRF2 protein expression (-), 1-4 weak positive (+), 5-8 positive $(++)$ and 9-12 strong positive (+++) expression. All IHC slides were reviewed independently by two investigators.

Cell culture and hormone treatment. Ovarian cancer cells, Hey, SKOV3, HO8910, HO8910PM, ES-2, OVCAR-3 and A2780 were obtained from the American Type Culture Collection (Manassas, VA, USA) and cultured in DMEM/F12 with 10\% fetal bovine serum (FBS), $100 \mathrm{U} / \mathrm{ml}$ penicillin, $100 \mu \mathrm{g} / \mathrm{ml}$ streptomycin, sodium pyruvate and L-glutamine in humidified $5 \% \mathrm{CO}_{2}$ at $37^{\circ} \mathrm{C}$. The Moody cell line was kindly provided by Dr W. Zheng (Arizona University, Tucson, AZ, USA), and is a normal ovarian epithelial cell line that was transfected with $h T E R T$. The cells were maintained in MCDB109/M199 medium supplemented with $15 \%$ FBS. When these cells reached $80 \%$ confluence, the cells were collected for western blot analysis to determine NRF2 expression in different cell lines.

To investigate the effect of hormones on NRF2 expression, Hey cells were plated in $10-\mathrm{cm}$ dishes with $1 \times 10^{5}$ cells $/ \mathrm{ml}$. After 24-h serum-starvation, the cells were treated with $50 \mathrm{mIU} / \mathrm{ml}$ follicle-stimulating hormone (FSH), $50 \mathrm{mIU} / \mathrm{ml}$ luteinizing hormone $(\mathrm{LH}), 1 \mathrm{nM} \beta$-estradiol $\left(\mathrm{E}_{2}\right)$ or $10 \mu \mathrm{M}$ medroxyprogesterone acetate (MPA) for $48 \mathrm{~h}$, respectively. Proteins were then extracted using standard methods for western blot assays.

ROS detection. Hey cells were plated onto glass slips in 6-well plates at $1 \times 10^{4}$ cells/well. After 24 -h serum-starvation, cells were treated with $50 \mathrm{mIU} / \mathrm{ml} \mathrm{FSH}, 50 \mathrm{mIU} / \mathrm{ml} \mathrm{LH}, 1 \mathrm{nM} \mathrm{E}{ }_{2}$ or $10 \mu \mathrm{M}$ MPA for $24 \mathrm{~h}$. For ROS determination, cells were incubated with dichlorofluorescein $(10 \mu \mathrm{g} / \mathrm{ml})$ for $30 \mathrm{~min}$ and were then fixed with $10 \%$ buffered formalin. Images were obtained with a fluorescence microscope.

Immunoblotting. All cells were harvested and lysed. Total protein extracts $(60 \mu \mathrm{g})$ were separated on $10 \%$ SDS-polyacrylamide gels and transferred to polyvinylidene fluoride (PVDF) membranes. Membranes were blocked with 5\% skimmed milk for $1 \mathrm{~h}$ and incubated overnight with anti-NRF2 primary antibody, followed by $1 \mathrm{~h}$ of incubation with the appropriate secondary antibody. Proteins were detected using an Enhanced Chemiluminescence reagent.

Statistical analyses. The statistical significance of intergroup differences was evaluated by the Fisher's exact test and the $\chi^{2}$ test. All statistical tests were two-sided and the signifi- 
A

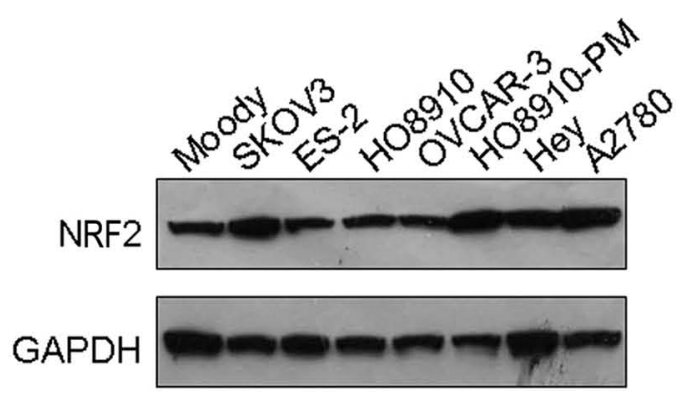

B
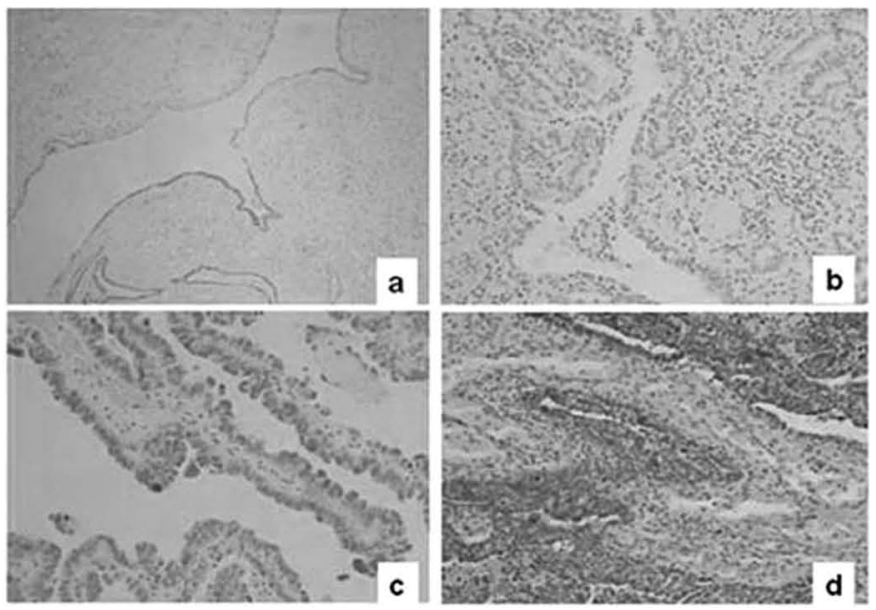

Figure 1. NRF2 protein expression in the ovarian surface epithelial cell line, ovarian cancer cell lines and ovarian tissues (cystadenoma, borderline tumor, high-grade serous carcinoma and low-grade serous carcinoma). (A) The expression pattern of NRF2 in ovarian surface epithelial cell line and ovarian cancer cell lines. (B) Immunohistochemical staining of benign ovarian cystadenomas tissues (a), borderline tumors (b), high-grade serous carcinoma (c) and low-grade serous carcinoma (d) tissues for NRF2 protein expression. Magnification, x100.

Table I. The expression of NRF2 in distinct tissue types.

\begin{tabular}{|c|c|c|c|c|c|c|c|}
\hline \multirow[b]{2}{*}{ Tissue type } & \multirow[b]{2}{*}{ Total } & \multicolumn{3}{|c|}{ Positive } & \multirow[b]{2}{*}{ Negative } & \multirow[b]{2}{*}{$\%$ positive } & \multirow[b]{2}{*}{ P-value } \\
\hline & & + & ++ & +++ & & & \\
\hline Cystadenomas & 10 & 3 & - & - & 7 & 30 & 0.006 \\
\hline Borderline tumor & 4 & - & - & - & 4 & 100 & \\
\hline Carcinomas & 64 & 13 & 10 & 19 & 22 & 65.6 & \\
\hline
\end{tabular}

Fisher's exact test, $\mathrm{P}=0.006$.

Table II. The expression of NRF2 in different OEC pathological types.

\begin{tabular}{lccccc}
\hline & & \multicolumn{2}{c}{ Expression } & & \\
\cline { 3 - 3 } Pathological type & Total & Positive & Negative & \% positive & P-value \\
\hline Clear cell carcinoma & 11 & 3 & 8 & 27.3 & 0.028 \\
Endometrioid adenocarcinoma & 9 & 7 & 2 & 77.8 & \\
Mucinous cystadenocarcinoma & 6 & 5 & 1 & 83.3 & \\
Serous cystadenocarcinoma & 38 & 27 & 11 & 71.1 & \\
\hline
\end{tabular}

$\chi^{2}$ test, $\mathrm{P}=0.028$.

cance level was established at 0.05 . All statistical analysis was performed using SPSS11.0 (SPSS Inc., Chicago, IL) or Prism 5.0 (GraphPad Software).

\section{Results}

Overexpression of NRF2 in ovarian epithelial carcinoma. As shown in Fig. 1A, NRF2 overexpressed in several ovarian cancer cell lines, including SKOV3, HO8910PM, Hey and A2780, when compared with normal ovarian epithelial Moody cell line. The expression of NRF2 in cystadenoma, borderline tumors and OEC cases was also evaluated.NRF2 was expressed in epithelial cells in 3/10 (30\%) benign ovarian cystadenoma samples (Table I); in these cases, very few positive cells were observed (Fig. 1Ba). Positive staining of NRF2 in borderline tumors was not observed (Fig. 1Bb). However, in ovarian carcinomas, NRF2 expression was enhanced in 44/64 (65.6\%) samples (Table I). Of these, 3/11 (27.3\%) clear cell carcinomas showed weak positive staining in the nucleus, $7 / 9(77.8 \%)$ endometrioid adenocarcinomas showed positive staining, 5/6 


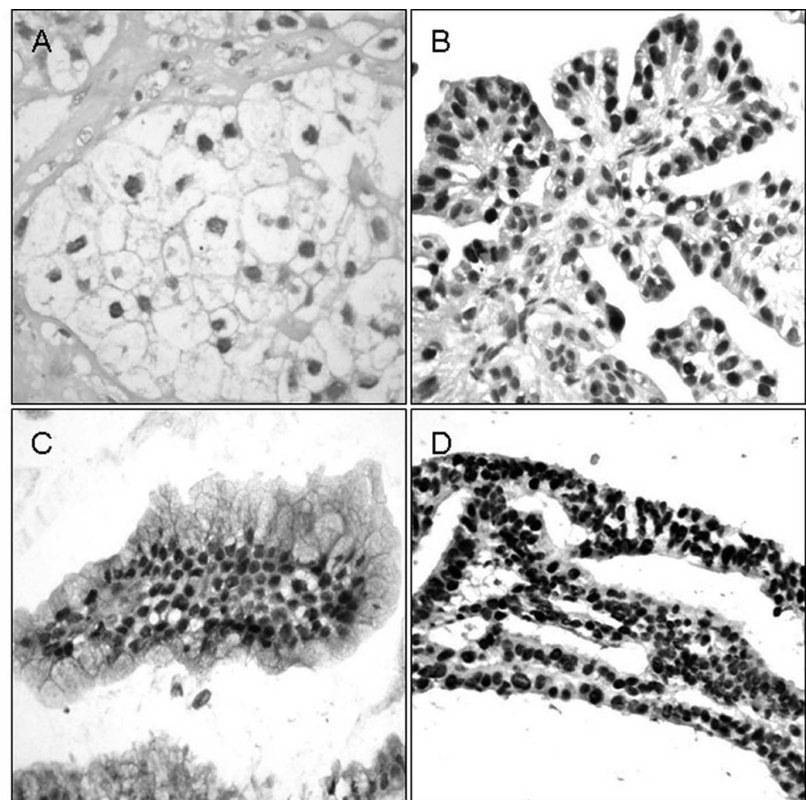

Figure 2. NRF2 protein expression in ovarian carcinoma of different pathological types. Immunohistochemical staining of clear cell carcinoma (A), endometrioid adenocarcinoma (B), mucinous cystadenocarcinoma (C) and serous cystadenocarcinoma (D) tissues for NRF2 protein expression. Magnification, $\mathrm{x} 400$.

(83.3\%) mucinous cystadenocarcinomas had positive nucleus staining, $27 / 38(71.1 \%)$ serous cystadenocarcinomas showed strong positive nucleus staining, and the remaining cases exhibited negative NRF2 expression (Table II). In these serous carcinomas cases, low-grade serous carcinomas shows an intensive positive NRF2 staining (Fig. 1Bd), whereas highgrade serous carcinomas shows relative weak positive staining (Fig. 1Bc). Compared with the cystadenoma samples, OEC samples showed an increased NRF2 expression $(\mathrm{P}=0.006)$. Representative pictures indicate low expression of NRF2 in cystadenoma samples (Fig. 1Ba) and strong positive staining in carcinomas (Fig. 1Bd). Although increased NRF2 expression in OEC was observed, the expression of NRF2 among different pathological types of tumor was variable. The majority of cases with high expression levels of NRF2 were serous cystadenocarcinomas (Fig. 3). Both endometrioid adenocarcinoma and mucinous cystadenocarcinoma also exhibited positive NRF2 protein expression; however, clear cell carcinoma showed a relatively low level of NRF2 expression $(\mathrm{P}=0.028)$ (Table II), compared with the other three pathological types. Representative pictures are shown in Fig. 2.

The relationship between prognosis and NRF2 expression. Table III shows the distribution of nuclear NRF2 positivity according to the clinicopathological characteristics of the malignant sample group. Within the age range of 22-79 years, $19 / 30(63.3 \%)$ cases in patients $>55$ years old and $23 / 34$ $(67.6 \%)$ cases $<55$ years old showed positive NRF2 staining. No significant correlation was observed between age and NRF2 expression.

The correlation between NRF2 expression and histological grade was also investigated. The malignant cases were classified into three groups according to the degree of differ-

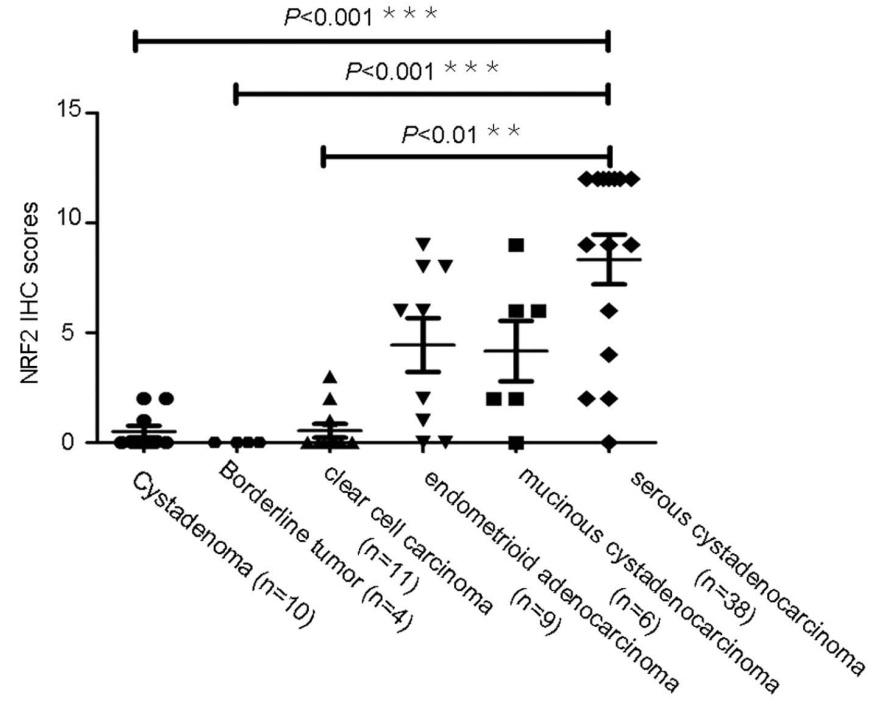

Figure 3. Comparison NRF2 protein expression among benign cystadenomas and different pathological types of ovarian carcinoma. *Statistically significant compared with serous cystadenocarcinoma $(\mathrm{P}<0.05)$.

entiation. Expression of NRF2 in ovarian carcinoma did not show statistically significant differences according to tumor grade (Table III). Similarly, no correlation between NRF2 expression and stage was observed.

Lymph node metastasis is an important prognostic factor for OEC, thus the relationship between NRF2 expression and lymph node metastasis was determined. NRF2 positive staining was detected in 21/33 (63.6\%) cases without lymph node metastasis, while 20/31 (64.5\%) cases with metastasis exhibited NRF2 expression (Table III). There was no correlation between NRF2 expression and lymph node metastasis.

The effects of hormones on ROS level in OEC. Gonadotrophins and sex-steroid hormones are high risk factors for OEC development. Increased NRF2 expression is generally accompanied by elevated ROS levels, so the ROS levels after hormone treatment were examined in Hey cells. Stimulation with $50 \mathrm{mIU} /$ $\mathrm{ml} \mathrm{FSH,} 50 \mathrm{mIU} / \mathrm{ml} \mathrm{LH}$ or $1 \mathrm{nM} \mathrm{E}_{2}$ resulted in increased ROS levels, as shown by increased green fluorescence (Fig. 4). Of these hormones, FSH had the greatest effect on ROS production. However, $10 \mu \mathrm{M}$ MPA treatment had no obvious effect on ROS production (Fig. 4).

The effects of hormones on NRF2 expression. We investigated the effects of hormones on NRF2 protein expression in ovarian cancer cells. FSH, LH, $\mathrm{E}_{2}$ and MPA significantly upregulated NRF2 expression, but by differing amounts, which suggests that NRF2 expression responds unequally to different hormonal stimuli (Fig. 5).

\section{Discussion}

NRF2 is a key transcriptional regulator of an intracellular antioxidant response through transcriptional activation of a battery of genes, including antioxidants and phase II detoxifying enzymes. NRF2 participates in multiple physiological processes, such as phase II drug metabolism and oxidative 
Table III. Correlations of NRF2 and major clinical pathological factors $(n=64)$.

\begin{tabular}{|c|c|c|c|c|}
\hline \multirow[b]{2}{*}{ Factors } & \multirow[b]{2}{*}{ No. of patients } & \multicolumn{2}{|c|}{ NRF2 expression } & \multirow[b]{2}{*}{ P-value } \\
\hline & & Positive & Negative & \\
\hline \multicolumn{5}{|l|}{ Age (years) } \\
\hline$<55$ & 34 & 23 & 11 & \multirow[t]{2}{*}{0.795} \\
\hline$\geq 55$ & 30 & 19 & 11 & \\
\hline \multicolumn{5}{|l|}{ Histological grade } \\
\hline Low (I) & 22 & 16 & 6 & \multirow[t]{3}{*}{0.531} \\
\hline Intermediate (II) & 18 & 12 & 6 & \\
\hline High (III) & 13 & 11 & 2 & \\
\hline \multicolumn{5}{|l|}{ Clinical stage } \\
\hline I & 13 & 7 & 6 & \multirow[t]{3}{*}{0.499} \\
\hline II & 16 & 10 & 6 & \\
\hline III & 35 & 25 & 10 & \\
\hline \multicolumn{5}{|c|}{ Lymph node metastasis } \\
\hline No & 33 & 21 & 12 & \multirow[t]{2}{*}{1.000} \\
\hline Yes & 31 & 20 & 11 & \\
\hline
\end{tabular}
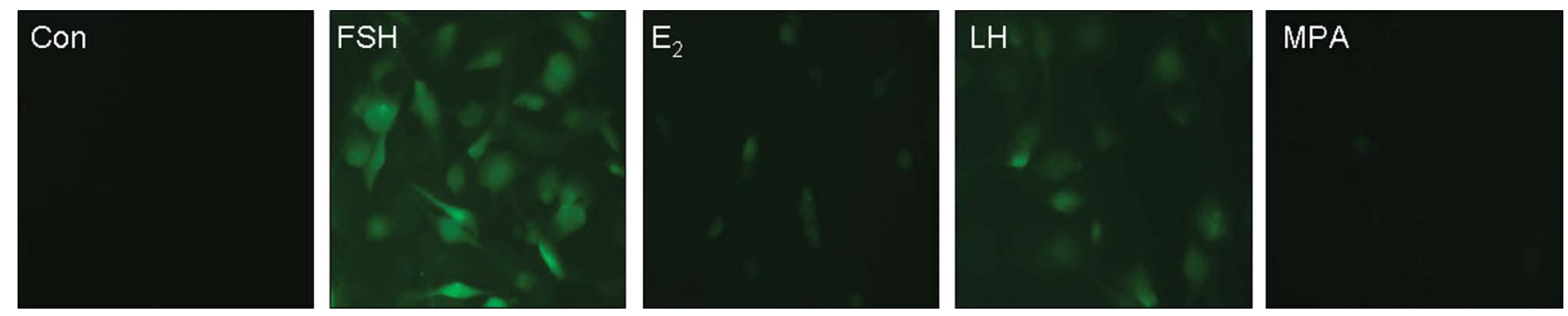

Figure 4. The effects of gonadotrophins and sex-steroid hormones on ROS production. Hey cells were plated onto a glass slip in the 6 -well plate at $1 \times 10^{4}$ cells/ well, after 24-h serum-starvation, the cells were treated with $50 \mathrm{mIU} / \mathrm{ml} \mathrm{FSH,} 50 \mathrm{mIU} / \mathrm{ml} \mathrm{LH}, 1 \mathrm{nM} \beta$-estradiol (E $\mathrm{E}_{2}$ ) and $10 \mu \mathrm{M}$ MPA for $24 \mathrm{~h}$, receptively. After incubation with dichlorofluorescein $(10 \mu \mathrm{g} / \mathrm{ml})$ for another $30 \mathrm{~min}$, the images were captured with a fluorescence microscope. Magnification, $\mathrm{x} 400$.

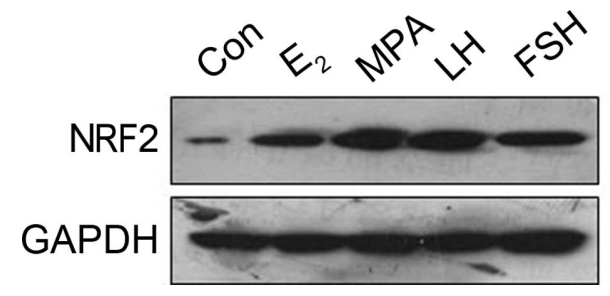

Figure 5. Gonadotrophins and sex-steroid hormones enhance NRF2 protein expression. Western blot analysis was used to determine NRF2 protein levels after treatment with $50 \mathrm{mIU} / \mathrm{ml} \mathrm{FSH}, 50 \mathrm{mIU} / \mathrm{ml} \mathrm{LH}, 1 \mathrm{nM} \beta$-estradiol $\left(\mathrm{E}_{2}\right)$ and $10 \mu \mathrm{M}$ MPA for $48 \mathrm{~h}$. GAPDH served as a loading control.

stress, and is negatively regulated by KEAP1. Aberrant NRF2 expression is found in various cancer types $(1,5-9)$ and the dysregulation of NRF2 affects cellular growth and response to therapy. Wang et al reported that overexpression of NRF2 in gallbladder adenocarcinoma was correlated with tumor differentiation, staging and metastasis (1). They also concluded that NRF2 was an independent prognostic factor, due to shorter overall survival times in patients with high levels of NRF2 expression (1). In addition, Chen et al reported that NRF2 was significantly highly expressed in endometrial serous carcinoma (ESC) and some endometrial precursor lesions, compared with atypical endometrial hyperplasia or endometrial intraepithelial neoplasia (3). Their study demonstrated that alteration of NRF2 expression may represent one of several early molecular events in ESC carcinogenesis. Similarly, Jiang et al showed that NRF2 is overexpressed in Type II endometrial cancer, and that this was correlated with resistance to chemotherapeutic drugs (2). Inhibition of NRF2 signaling may therefore increase the efficacy of chemotherapy, making NRF2 a potential target molecule for endometrial cancer therapy (2). However, information concerning NRF2 expression in OEC is scarce. In the current study, we observed that NRF2 overexpressed in several ovarian cancer cell lines. It was found that NRF2 expression was enhanced in $65.6 \%$ OEC cases, and was mainly localized to cell nuclei, by immunohistochemistry assay. Only a few weakly positive NRF2 staining cells could be observed in benign cystadenoma cases; however, a significant increase in NRF2 expression was exhibited in OEC cases. Further studies 
demonstrated that overexpression of NRF2 was more common in serous cystadenocarcinoma, endometrioid adenocarcinoma and mucinous cystadenocarcinoma than in clear-cell OEC. The lattermost pathological subtype showed increased NRF2 expression in only $27.3 \%$ of cases, which suggested that NRF2 expression was associated with pathological type in OEC. Although NRF2 is overexpressed in OEC, whether it could be used as an independent prognostic factor is not clear. In the present investigation, no relationships between NRF2 expression and patient age, tumor differentiation, staging or lymph node metastasis were found. In spite of being of limited use for prognostic assay, NRF2 expression may allow benign cystadenomas to be distinguished from malignant carcinomas. In addition, elevated NRF2 expression may be a molecular event in the process of OEC development; the reasons for increased NRF2 expression in OEC warrant further study.

Reactive oxygen species (ROS) play an important role in multiple normal physiological functions, such as ovulation (10). However, ROS are also involved in the development of cancer, due to induction of cellular DNA damage. In order to avoid the deleterious effects of excess ROS, NRF2 signaling is commonly activated by an antioxidant response that decreases ROS levels. However, if excess ROS cause constitutive activation of NRF2 signaling, the elevated NRF2 levels will result in chemoresistance, increased DNA repair, inflammatory responses and angiogenesis (11). ROS are commonly induced by a variety of external stimuli, including chemotherapeutics, growth factors and hormones (12-16). Generally, elevated ROS will induce a protective antioxidant response, enhancing the expression of NRF2. NRF2 in turn regulates an array of genes containing an enhancer sequence known as the ARE, which include glutamate cysteine ligase, heme oxygenase-1 (HO-1) and NAD(P)H quinine oxidoreductase-1 (NQ-1). However, the role of ROS production in response to gonadatrophins and sexsteroid hormones as high risk factors for OEC development has not been studied to date. In this study, we found that FSH, LH and estrogen (but not MPA) could induce ROS production at different levels. These hormones may therefore be involved in the cancer development process by inducing ROS.

Since some hormones are able to induce ROS production, they may also indirectly regulate NRF2 expression. To further determine the possible relationship between gonadotrophins, sex-steroid hormones and NRF2 expression, we treated Hey cells with $50 \mathrm{mIU} / \mathrm{ml} \mathrm{FSH}$ and $\mathrm{LH}, 1 \mathrm{nM} \mathrm{E_{2 }}$ and $10 \mu \mathrm{M}$ MPA. Our results indicate that FSH, LH, MPA and $\mathrm{E}_{2}$ cause the upregulation of NRF2 expression. FSH enhances OEC cell proliferation $(17,18)$, which may be mediated by elevated NRF2 levels, since NRF2 is implicated in the cellular growth of various cancers $(19,20)$. Ovulation is stimulated by the preovulatory surge of the pituitary LH, and a very recent report showed that LH-induced ROS is able to mimic the effect of LH and trigger ovulation (10). Under pathological conditions, LH may be involved in OEC development. It can therefore be speculated that LH-induced NRF2 may be associated with LH-mediated invasive and anti-apoptotic activity in OEC, but further studies to investigate this mechanism are required. In addition, excess estrogen exposure is a risk factor not only for $\mathrm{OEC}$ but also for breast cancer. Certain findings suggest that $\mathrm{E}_{2}$ promotes proliferation of ovarian epithelial cells. Studies done in the 1980s and 1990s of 'traditional' Asian women showed that their average serum $E_{2}$ level in the premenopausal period was $75-80 \%$ of that of US women (21). These lower $E_{2}$ levels may account for the lower ovarian cancer rates in Asian women. Although estrogen receptor (ER) positivity is found in 50-70\% of ovarian cancers (22), whether $\mathrm{E}_{2}$ enhances OEC development through ER signaling is still not clear. In breast carcinogenesis, some studies indicate that induction of ER-mediated signaling is not sufficient for the development of breast cancer and the underlying mechanisms of breast susceptibility to estrogen's carcinogenic effect may correlate with ROS induced by $\mathrm{E}_{2}(23)$. Here we showed elevated levels of NRF2 induced by $E_{2}$ which are associated with $\mathrm{E}_{2}$-induced ROS. Based on this finding, we suggest that $\mathrm{E}_{2}$-induced ROS and NRF2 are the molecular basis for OEC development. However, we did not observe this relationship between increased NRF2 expression induced by MPA and ROS levels induced by MPA. Therefore, we speculate that MPA upregulates NRF2 expression through another signaling mechanism, rather than ROS signaling.

This study denonstrated that NRF2 is overexpressed in human OEC tissues compared with benign cystadenomas, indicating that NRF2 may play a role in OEC development. This elevated NRF2 expression may be induced by gonadotrophins and sex-steroid hormones, which suggest that these hormones are involved in OEC development via modulation of NRF2 signaling. Therefore, the inhibition of NRF2 may represent an effective therapeutic strategy for the treatment of OEC.

\section{Acknowledgements}

The study was supported by grants from the National Natural Science Foundation of China (NSFC No. 81020108027 and No. 30872755 to Y.F.) grant from Science and Technology Commission of Shanghai Municipality (STCSM No. 09ZR1405000 to X.Y.) and supported by 200902 (to H.L.) from the Shanghai First Maternal and Fetal Care Hospital, Tongji University School of Medicine. The authors have no financial interest in this study.

\section{References}

1. Wang J, Zhang M, Zhang L, et al: Correlation of Nrf2, HO-1, and MRP3 in gallbladder cancer and their relationships to clinicopathologic features and survival. J Surg Res 164: 99-105, 2010.

2. Jiang T, Chen N, Zhao F, et al: High levels of Nrf2 determine chemoresistance in type II endometrial cancer. Cancer Res 70: 5486-5496, 2010.

3. Chen N, Yi X, Abushahin N, et al: Nrf2 expression in endometrial serous carcinomas and its precancers. Int J Clin Exp Pathol 4: 85-96, 2010 .

4. Wang XJ, Sun Z, Villeneuve NF, et al: Nrf2 enhances resistance of cancer cells to chemotherapeutic drugs, the dark side of Nrf2. Carcinogenesis 29: 1235-1243, 2008.

5. Akhdar H, Loyer P, Rauch C, Corlu A, Guillouzo A and Morel F: Involvement of Nrf2 activation in resistance to 5-fluorouracil in human colon cancer HT-29 cells. Eur J Cancer 45: 2219-2227, 2009.

6. Yao Y, Brodie AM, Davidson NE, Kensler TW and Zhou Q: Inhibition of estrogen signaling activates the NRF2 pathway in breast cancer. Breast Cancer Res Treat 124: 585-591, 2010.

7. Lister A, Nedjadi T, Kitteringham NR, et al: Nrf2 is overexpressed in pancreatic cancer: implications for cell proliferation and therapy. Mol Cancer 10: 37, 2011.

8. Fer ND, Shoemaker RH and Monks A: Adaphostin toxicity in a sensitive non-small cell lung cancer model is mediated through Nrf2 signaling and heme oxygenase 1. J Exp Clin Cancer Res 29: 91, 2010. 
9. Beyer TA, Auf dem Keller U, Braun S, Schafer M and Werner S: Roles and mechanisms of action of the Nrf2 transcription factor in skin morphogenesis, wound repair and skin cancer. Cell Death Differ 14: 1250-1254, 2007

10. Shkolnik K, Tadmor A, Ben-Dor S, Nevo N, Galiani D and Dekel N: Reactive oxygen species are indispensable in ovulation. Proc Natl Acad Sci USA 108: 1462-1467, 2011.

11. Kim TH, Hur EG, Kang SJ, et al: NRF2 blockade suppresses colon tumor angiogenesis by inhibiting hypoxia-induced activation of HIF-1 $\alpha$. Cancer Res 71: 2260-2275, 2011.

12. Bae YS, Kang SW, Seo MS, et al: Epidermal growth factor (EGF)-induced generation of hydrogen peroxide. Role in EGF receptor-mediated tyrosine phosphorylation. J Biol Chem 272 : 217-221, 1997.

13. Ohba M, Shibanuma M, Kuroki T and Nose K: Production of hydrogen peroxide by transforming growth factor- $\beta 1$ and its involvement in induction of egr-1 in mouse osteoblastic cells. J Cell Biol 126: 1079-1088, 1994.

14. Sundaresan M, Yu ZX, Ferrans VJ, et al: Regulation of reactive-oxygen-species generation in fibroblasts by Rac1. Biochem J 318: 379-382, 1996.

15. Sundaresan M, Yu ZX, Ferrans VJ, Irani K and Finkel T: Requirement for generation of $\mathrm{H}_{2} \mathrm{O}_{2}$ for platelet-derived growth factor signal transduction. Science 270: 296-299, 1995.

16. Kimura T, Okajima F, Sho K, Kobayashi I and Kondo Y: Thyrotropin-induced hydrogen peroxide production in FRTL-5 thyroid cells is mediated not by adenosine $3^{\prime}, 5^{\prime}$-monophosphate, but by $\mathrm{Ca} 2^{+}$signaling followed by phospholipase-A2 activation and potentiated by an adenosine derivative. Endocrinology 136 $116-123,1995$
17. Li Y, Ganta S, Cheng C, Craig R, Ganta RR and Freeman LC: FSH stimulates ovarian cancer cell growth by action on growth factor variant receptor. Mol Cell Endocrinol 267: 26-37, 2007.

18. Zheng W, Lu JJ, Luo F, et al: Ovarian epithelial tumor growth promotion by follicle-stimulating hormone and inhibition of the effect by luteinizing hormone. Gynecol Oncol 76: 80-88, 2000.

19. Gan N, Sun X and Song L: Activation of Nrf2 by microcystinLR provides advantages for liver cancer cell growth. Chem Res Toxicol 23: 1477-1484, 2010

20. Homma S, Ishii Y, Morishima Y, et al: Nrf2 enhances cell proliferation and resistance to anticancer drugs in human lung cancer. Clin Cancer Res 15: 3423-3432, 2009.

21. Wu AH and Pike MC: Dietary soy protein and hormonal status in females. Am J Clin Nutr 62: 151-153, 1995

22. Harding M, Cowan S, Hole D, et al: Estrogen and progesterone receptors in ovarian cancer. Cancer 65: 486-491, 1990.

23. Okoh V, Deoraj A and Roy D: Estrogen-induced reactive oxygen species-mediated signalings contribute to breast cancer. Biochim Biophys Acta 1815: 115-133, 2011. 